\title{
Synthesis and characterization of fluorescent carbon dots from tapioca
}

\begin{abstract}
This research demonstrates an economical and efficient reduction of carbon foot print. Tapioca powder as a source of organic carbon was utilized in the synthesis of carbon dots through optimization of the synthesis parameters such as temperature, dosage and time. Photoluminescent quantum yield (PLQY) was obtained under the visible region of $340 \mathrm{~nm}$ at $34.9 \%$, which was achieved without dopants such as sulfur and nitrogen that are popularly used to increase the value of photoluminescence. The characterization of carbon dots such as FTIR and HrTem, were carried out for the analysis of functional groups, particle sizes (1-5 $\mathrm{nm}$ ) and shapes (quasi-spherical). The high carbon-carbon bonds and oxygen groups detected in FTIR analysis validates the basis of fluorescence of carbon dots and also presence of hydroxyl $(\mathrm{OH})$, carboxylic acids $(\mathrm{COO})$, and other vital functional groups $(\mathrm{C}=\mathrm{O}, \mathrm{C}-\mathrm{O}-\mathrm{C}$, $\mathrm{C}-\mathrm{H})$. These characteristics makes tapioca based carbon dots suitable for application in the fields of environmental studies including sensitive detection and absorbance of pollutants in water and bio imaging in health sciences.
\end{abstract}

